\title{
ANÁLISE DA UTILIZAÇÃO DE DADOS DO SATÉLITE TRMM EM UM MODELO HIDROLÓGICO SEMIDISTRIBUÍDO NA BACIA DO RIO JAMARI (RO)
}

\author{
Ranyére Silva Nóbrega ${ }^{1}$ Enio Pereira de Souza ${ }^{2}$ e Francisco de Assis Salviano de Sousa ${ }^{3}$ \\ Artigo recebido em 20/08/2008 e aceito em 19/10/2008
}

\section{RESUMO}

Neste trabalho foi analisada a utilização de estimativas de precipitação geradas pelo satélite TRMM como variável de entrada em um modelo hidrológico semidistribuído, o Slurp. Para tal, foram utilizados dados diários de temperatura do ar, temperatura do ponto de orvalho e umidade relativa do ar, oriundos de estações meteorológicas; dados de cobertura do solo, derivados de imagens LandSat 5; dados fluviométricos diários e; dados do produto 3B42, com resolução temporal de 3 horas e espacial de $2,5 \mathrm{~km}$. Os resultados indicam que o modelo foi adequado ao simular a vazão na bacia do rio Jamari, com $\mathrm{R}^{2}$ de 0,82 . Com relação às vazões média, máxima e mínima, os resultados foram coerentes com de outros autores que utilizaram precipitação na superfície. O método de calibração do modelo mostrou-se hábil, mesmo na falta de alguns parâmetros, indicando que os resultados podem ser ainda melhores.

PALAVRAS-CHAVE: TRMM, modelagem hidrológica, Amazônia, Rondônia, Slurp

\section{DATA UTILIZATION ANALYSIS OF THE SATELLITE TRMM IN A SEMI- DISTRIBUITED HYDROLOGICAL MODEL IN THE WATERSHED JAMARI (RO)}

\section{ABSTRACT}

In this work was analyzed the precipitation estimate utilization generated by the satellite TRMM as input variable in a semi-distribuited hydrological model, Slurp. For such, were used diary data by air temperature, dew point temperature and relative humidity of the air, derived of meteorological stations; data of soil coverage, derivatives of images LandSat 5; runoff station data; 3B42 data product, with temporal resolution of 3 hours and spacial of 2,5 $\mathrm{km}$. The results indicate that the model was adequate to when simulating the flow in the river basin Jamari, with $\mathrm{R}^{2}$ of 0,82 . With regard to the average flows, maxim and minimum, the results were coherent with of other authors who used precipitation in the surface. The model calibration method it showed skilled, even in the lack of some parameters, indicating that the results can still be best.

KEY WORDS: TRMM, hydrological modeling, Amazon, Rondônia, Slurp

\footnotetext{
${ }^{1}$ Pesquisador do Sistema de Proteção da Amazônia - Centro Técnico e Operacional de Porto Velho. Av. Guaporé, 6056, Aeroporto. Porto Velho. RO. CEP: 78900-000. email: ranyere.nobrega@sipam.gov.br

${ }^{2}$ Professor Universidade Federal de Campina Grande - Unidade Acadêmica de Ciências Atmosféricas

${ }^{3}$ Professor Universidade Federal de Campina Grande - Unidade Acadêmica de Ciências Atmosféricas, e-mail: fassis@dca.ufcg.edu.br
} 


\section{INTRODUÇÃO}

A Floresta Amazônica é um dos mais importantes ecossistemas do planeta, com riquezas abundantes em vários aspectos como, por exemplo, recursos hídricos, fauna, flora, recursos minerais e étnico-culturais. Além disso, tem um importante papel no equilíbrio do planeta através dos ciclos hidrológico, carbono e energia.

O ciclo hidrológico pode ser considerado como um produto integrado entre o clima e os atributos biogeofísicos da superfície (Marengo, 2006). Gerar conhecimento sobre as componentes e variabilidade deste ciclo na bacia depende de conhecer os mecanismos físicos relacionados às forçantes que atuam neste ciclo, que se estendem de escala regionais a globais. Estes mecanismos são responsáveis por modular a variabilidade espacial e temporal hidrometeorológica na bacia, entre estas, a precipitação e a vazão.

Ao obter a precipitação em uma bacia hidrográfica pelos meios tradicionais (pluviômetros e pluviógrafos) as informações são pontuais, refletindo a chuva ocorrida em um dado ponto e, por vezes, é estendida para toda a bacia. Para ter um monitoramento detalhado sobre uma bacia, é necessária a existência de densa rede de instrumentos, que esbarra em problemas técnicos e de investimentos e, em se tratando de regiões como a Amazônica, também esbarra no difícil acesso em áreas de floresta e isoladas por rios.
A utilização de sensores remotos na estimativa de precipitação vem mostrando ser uma alternativa interessante para contrapor tais problemas. Nas últimas três décadas observaram-se avanços consideráveis no sensoriamento remoto no âmbito de satélites ambientais, aumentando a quantidade de informações disponíveis, incluindo estimativas de precipitação. Com melhores resultados nas estimativas os produtos têm migrado da pesquisa para o produto operacional, difundido por atores de hidrometeorologia (Nóbrega, 2008; Collischonn, 2006).

De fato, existem poucas informações sobre precipitação na bacia Amazônica, mas para a modelagem hidrometeorológica, não são as únicas necessárias, principalmente ao se utilizar modelos distribuídos. O modelo hidrológico semidistribuído Slurp vem mostrando ser uma alternativa interessante, uma vez que processa dados diretos de sensores remotos e não necessita de tantas informações e desempenho computacional quanto um modelo distribuído (Kite et al., 1999; Kite \& Droogers, 1999; Kite \& Granger, 2000; Kite 2001; Romero et al., 2002; Kite, 2005).

De acordo com o exposto acima, o objetivo deste trabalho é analisar o uso das estimativas de precipitação do satélite TRMM, em conjunto com o modelo hidrológico semidistribuído Slurp em uma bacia com poucas informações. O método também tem como base a utilização de dados que estão disponíveis gratuitamente, possibilitando gerar 
Revista Brasileira de Geografia Física

Recife-PE. V. 01, n. 01, Mai/Ago, 2008, 47-61

conhecimentos com pequeno custo operacional.

\section{ESTIMATIVA DE PRECIPITAÇÃO PELO TRMM: UMA REVISÃO}

A estimativa de chuva por satélite iniciou-se com o lançamento do primeiro satélite meteorológico, o TIROS I ("Televion and InfraRed Observation Satellite"). $\mathrm{Na}$ época, através das primeiras imagens de topos de nuvem (em abril de 1960), era possível estimar a ocorrência e até mesmo a intensidade de eventos chuvosos através da resposta espectral de conjuntos de nuvens (Petty, 1995). Nesta época, observaram que a radiação refletiva dos topos das nuvens poderia ser um indicador da sua espessura e conseqüentemente do volume de água.

O pioneiro em utilizar a técnica de indexação de nuvens foi Barret (1970). Até então as imagens de satélite não eram digitais. A metodologia é simples, baseia-se em assumir uma taxa de precipitação para cada tipo de nuvem. No trabalho do autor, ele objetivou estimar a precipitação mensal sobre a Austrália e suas vizinhanças associadas aos sistemas marítimos continentais. No final da década de 1970, as imagens passaram a ser digitais, melhorando consideravelmente a acurácia.

ARKIN (1979) desenvolveu um método de estimativa de precipitação no qual o autor usou o canal infravermelho do GOES para quantificar a relação entre cobertura de nuvens e temperatura do topo da nuvem para

quantificar o total de precipitação na superfície nos trópicos. Mais tarde este método ficou conhecido como GOES "Precipitation Index" (GPI) (Arkin \& Meisner, 1987), e ainda é bastante utilizado devido a sua simplicidade. A técnica baseia-se na alta correlação entre a fração de nuvens com temperaturas inferiores a $235 \mathrm{~K}(\sim-38 \mathrm{oC})$ e a área de chuva observada por radar em regiões de $2.5^{\circ} \times 2.5^{\circ}$ ao longo do mês.

Os primeiros métodos eram baseados em imagens nas bandas do visível e infravermelho. Após, surgiram metodologias utilizando imagens de sensores de microondas. Há uma tendência que as técnicas sejam aperfeiçoadas e expandidas para outros produtos, não só a precipitação.

No ano de 1997, os EUA em conjunto com o Japão lançaram um projeto denominado TRMM. O objetivo do projeto é medir a precipitação e a sua variação na região tropical a partir de satélites com órbita oblíqua nãoheliossíncrona de baixa inclinação e altitude. Os principais sensores a bordo do TRMM relacionados com a estimativa da precipitação são: TRMM “Microwave Imager" (TMI), "Precipitation Radar" (PR) e "Visible and Infrared Radiometer System" (VIRS), além destes há ainda os sensores para imageamento de relâmpagos (LIS) e sensor de energia radiante da superfície terrestre e das nuvens (CERES). A órbita baixa (inicialmente $350 \mathrm{~km}$, e após 2001 aproximadamente $403 \mathrm{~km}$ ) permite que o período de translação seja curto (91 minutos) permitindo resolução espacial e 
Revista Brasileira de Geografia Física

Recife-PE. V. 01, n. 01, Mai/Ago, 2008, 47-61

temporal relativamente alta.

Diversos estudos vêm sendo realizados para se obter medidas de precipitação para uma dada região, comparação com dados de superfície e dados de radar meteorológico, e até integração entre dados de superfície e de satélite.

SCHUMACHER \& HOUZE JR. (2000) compararam dados do TRMM com de um radar Doppler banda-S, que utiliza a equação Z/R para obtenção da precipitação. Os autores comentaram que a principal diferença ente os dois radares está na capacidade em capturar os campos de precipitação instantânea. Existe uma sensibilidade do radar PR que possui um limite de detectar taxas de precipitação maiores que $0,2-0,4 \mathrm{~mm} / \mathrm{h}$ (15-20 dBZ). Por causa deste limite de sensibilidade do PR, regiões com chuva estratiforme e eco associado com hidrometeoros de gelo no topo das nuvens são altamente subestimados. A combinação entre o radar banda-S e o PR podem resultar em estimativas mais precisas, uma vez que a freqüência do banda-S é ideal para detectar os hidrometeoros que o PR não consegue.

Estimativa de precipitação agrupada com dados de pluviômetros também tem sido utilizada. PEREIRA FILHO et al.(2002) compararam a técnica "Convective Stratiform Technique" (CST), derivada do satélite TRMM (sensor TMI), com estimativas de chuva a partir de radar e de uma rede de pluviômetros em São Paulo. $\mathrm{Na}$ área de cobertura do radar $(\sim 300 \mathrm{~km} 2)$, foram comparadas estimativas de chuva diárias, nos anos de 2001 e 2002 distribuídas em "grid” de $4 \times 4 \mathrm{~km}^{2}$. Segundo o autor nenhum dos três instrumentos são efetivos na representação da alta variabilidade espacial e temporal da precipitação, quando analisados separadamente. $\mathrm{O}$ autor recomenda técnicas estatísticas para integrar os três instrumentos.

NICHOLSON et al. (2003) validou o TRMM com uma densa rede de estações no oeste africano na fase pré-TRMM quando os sensores e algoritmos ainda estavam sendo testados. Os autores encontram correlação de 0,92 para o ano de 1998 quando comparado a ocorrência de chuva, no entanto, quantitativamente encontraram erros entre $20 \mathrm{e}$ $40 \%$. Concluíram que o sensor utilizado responde a variação de chuva, mas quantifica incorretamente os valores.

FEKETE et al. (2004), testaram seis diferentes conjuntos de dados de precipitação em um modelo simples de balanço hídrico global, que calcula a vazão média de longo período através da diferença entre precipitação e evapotranspiração. Foram utilizados dados de sensores remotos, postos pluviométricos e de reanálises, entre estes, dados do satélite TRMM. Os autores obtiveram resultados muito similares entre os diversos conjuntos de dados, mostrando que as estimativas de satélite podem ser dados relativamente confiáveis. No entanto, é importante ressaltar que o modelo utilizado é excessivamente simplificado, além disso, o estudo foi no contexto global e com dados mensais. 
Revista Brasileira de Geografia Física

Recife-PE. V. 01, n. 01, Mai/Ago, 2008, 47-61

COLLINSCHON et al. (2007), $62^{\circ} 36^{\prime} \mathrm{W}$ a $64^{\circ} 20^{\prime} \mathrm{W}$ longitude. A escolha foi

COLLINSCHON et al. (2006) e RAMAGE et

al. (2003) encontraram erros relativos de $9 \%$

para a precipitação acumulada anual comparado com dados de superfície. Valores semelhantes foram obtidos por Nóbrega et al. (2008a,b) comparando os dados do TRMM, com os "Climate Prediction Center" (CPC) e quando comparada com dados de estações meteorológicas o erro relativo foi de $5 \%$. O sensor de microondas do TRMM também foi utilizado para gerar uma série de dados climáticos diários por Layberry et al. (2006) na África.

Para fins de modelagem hidrológica, um dos precursores no Brasil foi o trabalho do Collischonn (2006) que utilizou os dados do TRMM com o modelo MGB para analisar sua funcionalidade na hidrometeorologia sobre a bacia do rio São Francisco até Três Marias. O autor concluiu que a ferramenta pode ser utilizada, resultando em bons resultados nas simulações hidrológicas, além disso, os dados podem ajudar a identificar pluviômetros com problemas na leitura ou mal localizados, constituindo-se em ferramenta para consistência de dados.

\section{MATERIAL E MÉTODOS}

\section{Área de estudo}

Para o estudo foi escolhida a bacia do rio Jamari localizada no estado de Rondônia (Figura 1) delimitada pelas coordenadas geográficas $08^{\circ} 28^{\prime} \mathrm{S}$ a $11^{\circ} 07^{\prime} \mathrm{S}$ de latitude e estratégica mediante dois fatores: importância e tamanho.

A bacia deságua em outra bacia maior, a do rio Madeira; o rio Madeira é um dos mais importantes afluentes do rio Amazonas. O rio Jamari tem sua nascente no sudoeste da Serra dos Pacaás Novos, em Rondônia e seu curso tem direção no sentido norte, desembocando na margem direita do rio Madeira. Possui uma área aproximada de 29.066,68 km² (Fonte: Eletronorte). Seus principais afluentes pela margem direita são: rio Branco, Preto do Crespo e Quatro Cachoeiras e, pela margem esquerda, os rios Massangana e Candeias.

Com relação aos recursos hídricos, a bacia do rio Jamari tem grande significado econômico para Rondônia por ter sido represada para a formação da primeira usina hidrelétrica do Estado, a Usina Hidrelétrica Samuel, e servir como importante via de transporte de passageiros e cargas na região compreendida entre os municípios de Porto Velho e Ariquemes.

A presença de uma rede urbana articulada em torno do eixo rodoviário da BR364 e tendo como centros polarizadores a capital Porto Velho e o centro sub-regional de Ariquemes estrutura os fluxos econômicos desta bacia a partir dos quais se projetam, dinamicamente, novas formas de apropriação e uso do território compreendido pela bacia do Jamari.

Com relação ao tamanho, por ser menor do que outras da região, demanda 
Revista Brasileira de Geografia Física

Recife-PE. V. 01, n. 01, Mai/Ago, 2008, 47-61

menos tarefa computacional, podendo ser informações. A resolução horizontal é de 90 utilizada como bacia-piloto da metodologia proposta neste trabalho.

\section{Dados utilizados}

O modelo Slurp necessita de três tipos de dados primários: dados de elevação digital (DEM), dados climáticos (depende do método de obtenção da evapotranspiração) e dados de cobertura do solo.

\section{Dados de elevação digital}

Para os DEM's foram utilizados os dados da missão "Shuttle Radar Toopgraphy Mission" (SRTM) realizada em parceria com o "Jet Propulsion Laboratory" (JPL) da NASA, "National Imagine \& Main Agency" (NIMA) do Departamento de Defesa dos Estados Unidos (DOD) e as Agências Espaciais Alemã e Italiana.

O DEM é um modelo representativo dos valores da altitude em todos os pontos numa determinada região. Pode ser expresso também por uma função matemática de interpolação no espaço vetorial $\mathrm{R}^{2}$ ou $\mathrm{R}^{3}$ (RABUS et al., 2003; GALVÍNCIO \& SOUSA, 2004; GALVÍNCIO, 2005). No entanto, por se tratarem de dados remotos, estão sujeitos a uma série de fatores que alteram a relação da veracidade para com a verdade terrestre. Seu uso, portanto, requer o desenvolvimento de processos de prétratamento, para que atendam, ou se aproximem, à demanda técnica da modelagem de relevo e sua integração com outras

metros e as falhas existentes foram preenchidas seguindo a metodologia proposta por NÓBREGA et al. (2005).

\section{Dados de cobertura do solo}

Os dados de cobertura do solo foram derivados de imagens LandSat 5 do ano de 2006, com resolução de 30 metros, cedidas pelo Sistema de Proteção da Amazônia (Sipam). Para a área da bacia foram utilizadas sete cenas. As imagens foram georeferenciadas utilizando como base os dados Geocover (http://glcf.umiacs.umd.edu/portal/geocover/).

Após esse procedimento foi construído o mosaico das sete cenas.

No processo de derivação dos dados de cobertura/uso do solo foi utilizada a metodologia descrita em KITE (2005), que consiste basicamente em obter o Índice de Vegetação Normalizada (NDVI) e fazer uma classificação dos dados. Neste caso foi usada uma classificação não-supervisionada, com quatro classes: floresta, pastagem, antropizada e água. Na classe antropizada estão incluídas, áreas degradadas e áreas urbanas (maiores detalhes podem ser obtidos em NÓBREGA, 2008).

A matriz dos dados de cobertura do solo precisa ser do mesmo tamanho da matriz do DEM, por isso, os dados tiverem que ser reamostrados para uma escala de 90 metros. Por fim, foi necessário converter o arquivo de cobertura do solo para o formato texto para que o modelo hidrológico possa acessar; este 
Revista Brasileira de Geografia Física

Recife-PE. V. 01, n. 01, Mai/Ago, 2008, 47-61

procedimento foi realizado em primeira etapa

com o Erdas Imagine e em segunda etapa com um programa desenvolvido em Fortran 4.0.

\section{Dados climáticos}

Foram utilizados dados diários de temperatura do ar, temperatura do ponto de orvalho e umidade relativa do ar, disponibilizados pela Secretaria de Desenvolvimento Ambiental do Estado de Rondônia (Sedam) de quatro estações meteorológicas (Ariquemes, Porto Velho, Cacoal e Ji-Paraná), para o período de 1999 a 2006.

\section{Dados de estimativa de precipitação}

Para a precipitação foram utilizados dados do algoritmo 3B42 do satélite TRMM. $\mathrm{O}$ produto $3 \mathrm{~B} 42$ gera estimativas de precipitação de 3 em 3 horas com resolução de $0,25^{\circ} \times 0,25^{\circ}$ graus.

Os dados são gravados em formato TRMM HDF e podem ser lidos com o software proprietário TSDIS Orbit Viewer ( ftp://disc2.nascom.nasa.gov/software/trmm_s oftware/Orbit_Viewer). Durante o trabalho foram testados alguns softwares de geoprocessamento para leitura dos dados (ArcGis, Erdas, Idrisi, Surfer e GlobalMapper), o único que conseguiu abrir os arquivos foi o Idrisi, mas com limitações, como imagens rotacionadas em $90^{\circ}$. Os dados podem acessados no endereço http://disc.sci.gsfc.nasa.gov/data/datapool/TR MM_DP/01_Data_Products/02_Gridded/06_3-
hour_Gpi_Cal_3B 42/

Atualmente a NASA está disponibilizando os dados em formato binário, que permitem serem lidos no GrADS ("Grid and Analysis Display System”). A leitura no GrADS facilita do ponto de vista que é uma ferramenta que aceita automatização de tarefas através de "Grads Script" (GS).

Foram desenvolvidos dois GS: i) para obter a precipitação diária do TRMM e; ii) para converter os dados binários para texto no formato ano, mês, dia, precipitação.

A resolução espacial do TRMM permitiu a utilização de 68 pontos com informações de precipitação dentro e nas proximidades da bacia do rio Jamari.

\section{Dados fluviométricos}

Na região de estudo há apenas a estação fluviométrica de Santa Isabel em operação com dados confiáveis, segundo a Eletronorte, pertencente a ANA/Sipam. Há diversas outras estações na bacia, mas a grande maioria estão atualmente inativas.

\section{Paramêtros para o Slurp}

O Slurp necessita de outros parâmetros como índice de Vegetação da Diferença Normalizada (NDVI); índice de área foliar (IAF), albedo tipo de solo. Estes parâmetros foram obtidos através de consulta literária (NÓBREGA, 2008). Parâmetros como capacidade de armazenamento de água no solo foram obtidos de através da calibração do modelo. 


\section{Período de estudo}

Os dados do TRMM estão disponíveis de outubro de 1998 até a atualidade, assim como a maioria dos dados climáticos das estações utilizadas (algumas estão disponíveis desde o início de 1998). No entanto, para este estudo foram utilizados os anos de 1999 a 2006, excluindo 2007, haja vista os dados da estação fluviométrica não estavam consistidos até a execução do trabalho.

No processo de calibração foram utilizados os anos de 01/01/1999 a 31/12/2003 e para verificação 01/01/2004 a 31/12/2006.

\section{Calibração}

O modelo pode ser calibrado de três modos diferentes:

1. Manualmente, através de mudanças individuais dos parâmetros, fazendo uma série de simulações e comparando os resultados gerados;

2. Método "Shuffled Complex Evolution" (SCE-UA) desenvolvido na Universidade do Arizona (Duan et al., 1994);

3. Prepando arquivos para o uso do método PEST (WATERMARK NUMERICAL COMPUTING, 2000), sendo que este método é independente do Slurp, funcionando como um módulo adicional.

Neste trabalho foi utilizado o método SCE-UA que é baseado em algoritmos genéticos e está presente dentro do modelo Slurp. O SCE-UA é um algoritmo de calibração automática mono-objetivo desenvolvido na Universidade do Arizona (DUAN et al., 1994) para otimização de funções e calibração de parâmetros de modelos. Este algoritmo combina técnicas de algoritmos genéticos e de busca direta pelo método Simplex de Nelder e Mead.

Ele opera com uma população de pontos que "evolem" em direção ao ótimo global através de sucessivas iterações e avaliações da função objetivo. Cada um destes pontos é definido pelos valores dos parâmetros e é um candidato ao ótimo (COLLISCHONN, 2001).

\section{Critérios para Avaliação}

Apesar da avaliação das simulações poderem ser realizadas de modo qualitativo, comparando os hidrogramas simulados e calculados, tem-se adotado na literatura o uso de coeficientes de avaliação (também conhecidos por função-objeto). Dentro os diversos coeficientes existentes, aqui foram utilizados como critério de avaliação das simulações o Coeficiente de Nash-Sutcliffe $\left(R^{2}\right)$

O valor de $R^{2}$ é dado por:

$$
R^{2}=\frac{F_{m}^{2}-F_{d}^{2}}{F_{m}^{2}}
$$

em que

$$
F_{m}^{2}=\frac{1}{n} \sum_{i=1}^{n}\left(q_{i}-\bar{q}\right)^{2}
$$

e 
Revista Brasileira de Geografia Física

Recife-PE. V. 01, n. 01, Mai/Ago, 2008, 47-61

$$
F_{d}^{2}=\frac{1}{n} \sum_{i=1}^{n}\left(q_{i}-c_{i}\right)^{2}
$$

$n$ é o número de dias, $q_{i}$ é o fluxo observado no dia $i, c_{i}$ é o fluxo simulado no dia $i$, e $\bar{q}$ é o fluxo médio.

$\mathrm{O} \mathrm{R}^{2}$ representa a parcela da variância dos dados que é explicada pelo modelo, podendo variar de $-\infty$ a 1 . Segundo O. SILVA et al. (2008) e COLLINSCHONN (2001) o desempenho de um modelo é considerado adequado e bom se o valor de $\mathrm{R}^{2}$ for maior que 0,75 , considerado aceitável se $\mathrm{R}^{2}$ for entre 0,36 e 0,75 , e inaceitável se for inferior a 0,36 .

\section{RESULTADOS E DISSCUSSÃO}

Ao executar o modelo sem calibração a vazão média anual obtida foi de $746,74 \mathrm{~m}^{3} / \mathrm{s}$, com coeficiente de Nash-Sutcliffe $\left(\mathrm{R}^{2}\right)$ de 2,94. Os resultados podem ser observados na Figura 2, na qual apresenta o hidrograma de vazão diária para o período de 1999 a 2006 na bacia do rio Jamari, em Rondônia, para o experimento TRMM sem calibração. O modelo não conseguiu representar bem a vazão, o que era esperado, uma vez que ainda não havia sido realizada a calibração.

Após a calibração do modelo a vazão calculada foi de $795,52 \mathrm{~m}^{3} / \mathrm{s}$, com $\mathrm{R}^{2}$ de 0,82 . Observando os valores, nota-se uma melhora do $\mathrm{R}^{2}$ indicando a eficiência do modelo após o processo de calibração. COLLINSCHON (2006) encontrou resultados semelhantes com relação à comparação entre dados do TRMM e uma densa rede de pluviômetros na bacia do

São Francisco até a Usina de Três Marias.

$O$ coeficiente $R^{2}$ pode variar a partir de negativo infinito até 1 , sendo 1 um ajuste perfeito. $\mathrm{O}$ valor de $\mathrm{R}^{2}$ é influenciado por erros nas vazões máximas, razão por que, quando $\mathrm{R}^{2}$ é próximo de 1 , o modelo está obtendo bom ajuste para as cheias.

Observa-se na Figura 3 que a qualidade da calibração é muito boa. Alguns picos de vazão estão bem representados, em outros o modelo chegou próximo, mas sempre acompanhando a tendência sazonal. Nos períodos de vazante observa-se superestimativa, já nos períodos de cheia subestimativa. É possível que este resultado se deva aos valores de capacidade de armazenamento do solo, que foram derivados de forma automática através da calibração.

Com relação às vazões máximas e mínimas, o modelo calculou vazão máxima de $2045,6 \mathrm{~m}^{3} / \mathrm{s}$ (e a observada foi de 2483,99 $\mathrm{m}^{3} / \mathrm{s}$ ), indicando subestimação da vazão máxima. A vazão mínima calculada foi de $127,6 \mathrm{~m}^{3} / \mathrm{s}$, superestimando a mesma (a observada foi de $86,76 \mathrm{~m}^{3} / \mathrm{s}$ ). Os resultados foram coerentes com os obtidos por RIBEIRO NETO (2006) para a bacia do rio Machado, no entanto o autor utilizou dados de precipitação observados.

Os resultados para a vazão média mensal podem ser observados na Figura 4, a qual apresenta o hidrograma da vazão média mensal para o período em estudo. O modelo 


\section{Revista Brasileira de Geografia Física}

\section{Recife-PE. V. 01, n. 01, Mai/Ago, 2008, 47-61}

tende a obter melhor resultado durante $\mathrm{o}$ período de cheia, mas subestima o pico (apesar de acompanhar a tendência sazonal). No período de vazante o modelo tende a superestimar os dados observados. Os dados do TRMM mostraram bons resultados na simulação média mensal.

Os resultados indicam que o TRMM é uma boa ferramenta para a hidrometeorologia. Pode se tornar mais importante ainda quando há falta de informações pluviométricas em superfície. No caso de Rondônia, apesar de existir 19 estações meteorológicas e 17 postos pluviométricos (entre ANA/Sipam, Inmet, Sedam e outros órgãos estaduais), há problemas no recebimento dos dados. Das estações meteorológicas, apenas em 3 os dados são transmitidos diariamente, nas outras as transferências dos dados ainda é realizada por módulo, além disso, há também estações com problemas técnicos. Já os dados da ANA/Sipam, precisam passar por consistência, estando sujeitos também há atrasos que podem chegar a dias para o recebimento dos dados. O TRMM disponibiliza as informações em praticamente tempo real (através do produto 3B42RT), com atrasos de menos de 2 horas, permitindo tomada de decisões mais ágeis no que tange o gerenciamento de recursos hídricos.

\section{CONCLUSÕES}

1. De acordo com os resultados obtidos espera-se que esta metodologia possa ser aplicada em outras bacias da região. Uma das principais características desta metodologia é utilizar um modelo hidrológico semidistribuído que utiliza dados de sensores remotos e não precisar de uma tarefa computacional tão árdua quanto em um modelo distribuído. Outra característica é a utilização direta de dados de sensores remotos na modelagem hidrológica, dados estes que estão disponíveis em tempo real pelo satélite TRMM, suprindo a falta de informações sobre a região estudada.

2. O método de calibração SCE-UA mostrou-se eficiente, mesmo na ausência de informações como a capacidade de armazenamento de água no solo, por exemplo, indicando que os resultados podem ser ainda melhores.

3. O coeficiente de verificação indica que o modelo é adequado para a utilização, com $\mathrm{R}^{2}(0,82)>0,75$.

\section{AGRADECIMENTOS}

Nós agradecemos ao Sistema de Proteção da Amazônia (Sipam) pelas imagens LandSat e dados fluviométricos; a Sedam pelos dados climáticos; ao Bruno Collinschonn pelo suporte com o TRMM; ao CNPq/ProSul pelo incentivo financeiro à pesquisa.

\section{REFERÊNCIAS}

ARKIN, P.A. \& MEISNER, B.N. The relationship between large-scale convective rainfall and cold cloud over the Western Hemisphere during 1982-84. Mon. Wea. Rev., 115: 51-74. 1987. 
BARRETT, E. C. The estimation of monthly rainfall from satellite data. Mon. Wea. Rev., 98: 322-327. 1970.

COLLISCHONN, B. Uso de precipitação estimada pelo satélite TRMM em modelo hidrológico distribuído. Dissertação de Mestrado. Universidade Federal do Rio Grande do Sul. 2006.

COLLISCHONN, B.; ALLASIA, D.; COLLISCHONN, W.; TUCCI, C.E.M. Desempenho do satélite TRMM na estimativa de precipitação sobre a bacia do Paraguai superior. Revista Brasileira de Cartografia, 59. 2007.

COLLISCHONN, W. e TUCCI, C.E.M. Simulação hidrológica de grandes bacias. Rev. Bras. de Rec. Hid.,6 (1): 95-118. 2001.

DUAN, Q. SOROOSHIAN, S. GUPTA, V.K. Optimal use of the SCE-UA global optimization method for calibrating watershed models. Journ.of Hydrol. 158: 265-284. 1994.

FEKETE, B.M.; VÖRÖSMARTY, C.J. et al. Uncertainties in precipitation and their impacts on runoff estimatites. Journ. of Clim. 17: 294304. 2004.

GALVÍNCIO, J. D.; SOUSA F. A. S. Uso do modelo TOPAZ para a caracterização fisiográfica da bacia hidrográfica do Açude Epitácio Pessoa. Rev. Bras. de Rec. Hid. 9 (4): 120-139, 2004.

GALVÍNCIO, J.D. Balanço Hídrico à superfície da Bacia Hidrográfica do Açude Epitácio Pessoa. Campina Grande, PB: UFCG. 2005. 153p. Tese de Doutorado. Universidade Federal de Campina Grande, 2005.

KITE, G. Manual for the SLURP hydrological model. 2005.

KITE, G.W., DROOGERS, P.; MURRAYRUST, H.; De VOOGT, K. Modeling scenarios for water allocation in the Gediz Basin, Turkey. Research Report 50,
International Water Management Institute, Colombo, Sri Lanka. 1999.

KITE, G.W.; DROOGERS, P. Integrated basin modeling. Research Report 43, International Water Management Institute, Colombo, Sri Lanka. 1999.

KITE, G.W.; GRANGER, R. Evapotranspiration at the basin scale estimated from satellite and hydrological model. Water Resources Bulletin, IAHS, Wallingford. 2000 .

MARENGO, J. A. On the hydrological cycle of the Amazon basin: a historical review and current state-of-the-art. Rev. Bras. Meteorol. 21 (3). 2006.

NICHOLSON, S.E.; et. al. Validation of TRMM and Other Rainfall Estimates with a High-Density Gauge Dataset for West Africa. Part II: Validation of TRMM Rainfall Products. J. Appl. Meteor., 42: 1355-1368. 2003.

NÓBREGA, R.N. Modelagem de Impactos do Desmatamento nos Recursos Hídricos da Bacia do Rio Jamari (Ro) utilizando dados de Superfície e do TRMM. Campina Grande, PB: UFCG. 2008. 238 pág. Tese de Doutorado. Universidade Federal de Campina Grande, 2008 .

NÓBREGA, R.S.; SILVA, D. F.; ARAÚJO, L.E.; SOUZA, F.A.S. Método para filtragem de Modelo de Elevação Digital (SRTM): análise para a Sub-bacia do rio Espinharas (PB). Anais... In: SIMPÓSIO INTERNACIONAL DE CLIMATOLOGIA, 2005, Fortaleza, 2005.

O. SILVA, P.M.; MELLO, C.R. de; SILVA, A.M. da; COELHO, G. Modelagem da hidrógrafa de cheia em uma bacia hidrográfica da região Alto Rio Grande. Rev. bras. Eng. agríc. ambient. 12(3). 2008.

PEREIRA FILHO, A. J.; NEGRI, A.; NAKAYAMA, P.T. An inter-comparison of gauge, radar and satellite rainfall in the tropics. 1st International Precipitation Working Group 
Workshop, CGMS-WMO, Proceedings, 275284, Madrid, Spain, 2002.

PETTY, G.W. Physical retrievals of overocean rain rate from multichannel microwave imager. Part I: Theoretical characteristics of normalized polarizations and scattering indices. Meteorology and Atmospheric Physics.54: 79-99, 1994.

RABUS, B; EINEDER, M; ROTH, A. The shuttle radar topography mission--a new class of digital elevationmodels acquired by spaceborne radar. ISPRS Journal of Photogrammetry and Remote Sensing 57(4): 241-262. 2003.

RAMAGE, K.; JOBARD, I.; LEBEL, T.; DESBOIS, M. Satellite estimation of 1-day to 10-day cumulated precipitation: comparison and validation over tropical Africa of TRMM, METEOSAT and GPCP products. IN: The 2003 EUMETSAT Meterological Satellite Conference. Weimar, Alemanha. 2003.
RIBEIRO NETO, A. Simulação Hidrológica na Amazônia: Rio Madeira. Tese de Doutorado. Universidade Federal do Rio de Janeiro. 178 páginas. 2006.

ROMERO,D.; MADRAMOOTOO, C.A., ENRIGHT, P. Modelling the hydrology of an agricultural watersehd in Quebec using SLURP. Canadian Biosystems Engineering. 44: 1.11-1.20. 2002.

SCHUMACHER, C., HOUZE JR.; R.A. Comparison of radar data from the TRMM satellite and Kwajalein oceanic validation site. J. Appl. Meteor. 39: 2151-2164. 2000.

TRMM. Mission Overview. Disponível em $<$ http://trmm.gsfc.nasa.gov $>$. Acesso em setembro de 2006.

WATERMARK

NUMERICAL

COMPUTING. PEST, model-independent parameter estimation. 2000.

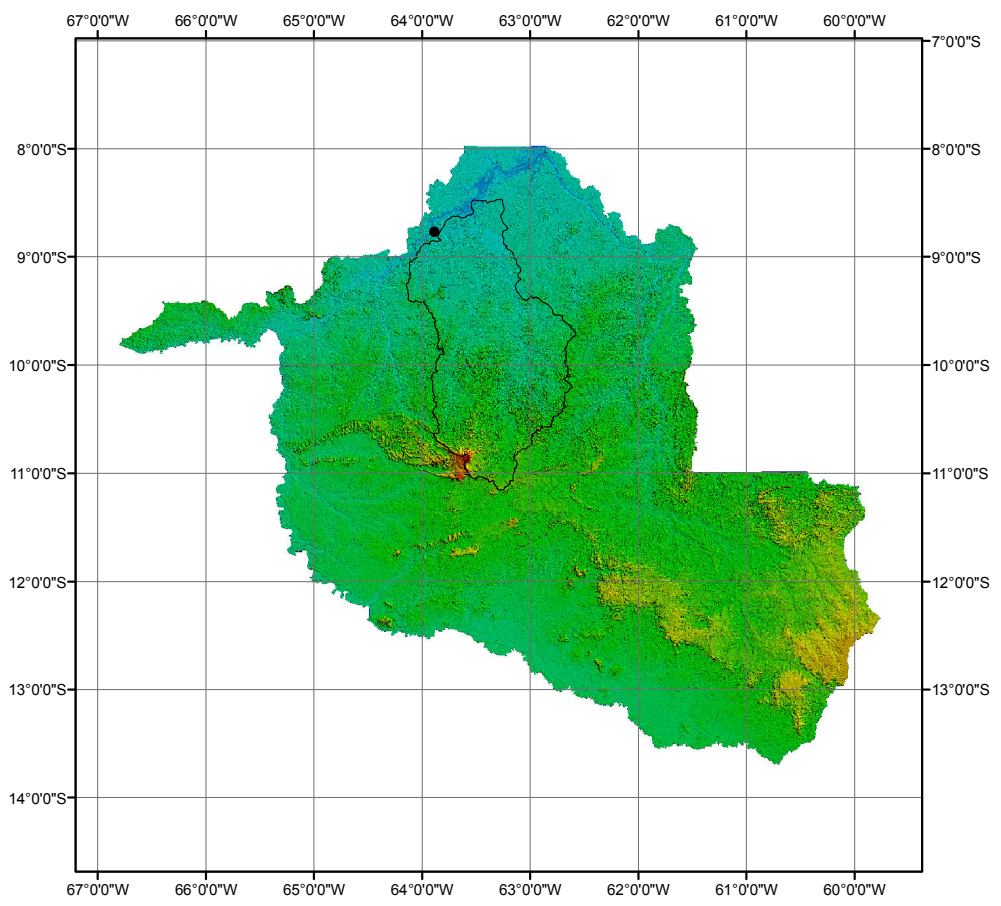

Figura 1 - Localização geográfica da bacia do rio Jamari 
Revista Brasileira de Geografia Física

Recife-PE. V. 01, n. 01, Mai/Ago, 2008, 47-61

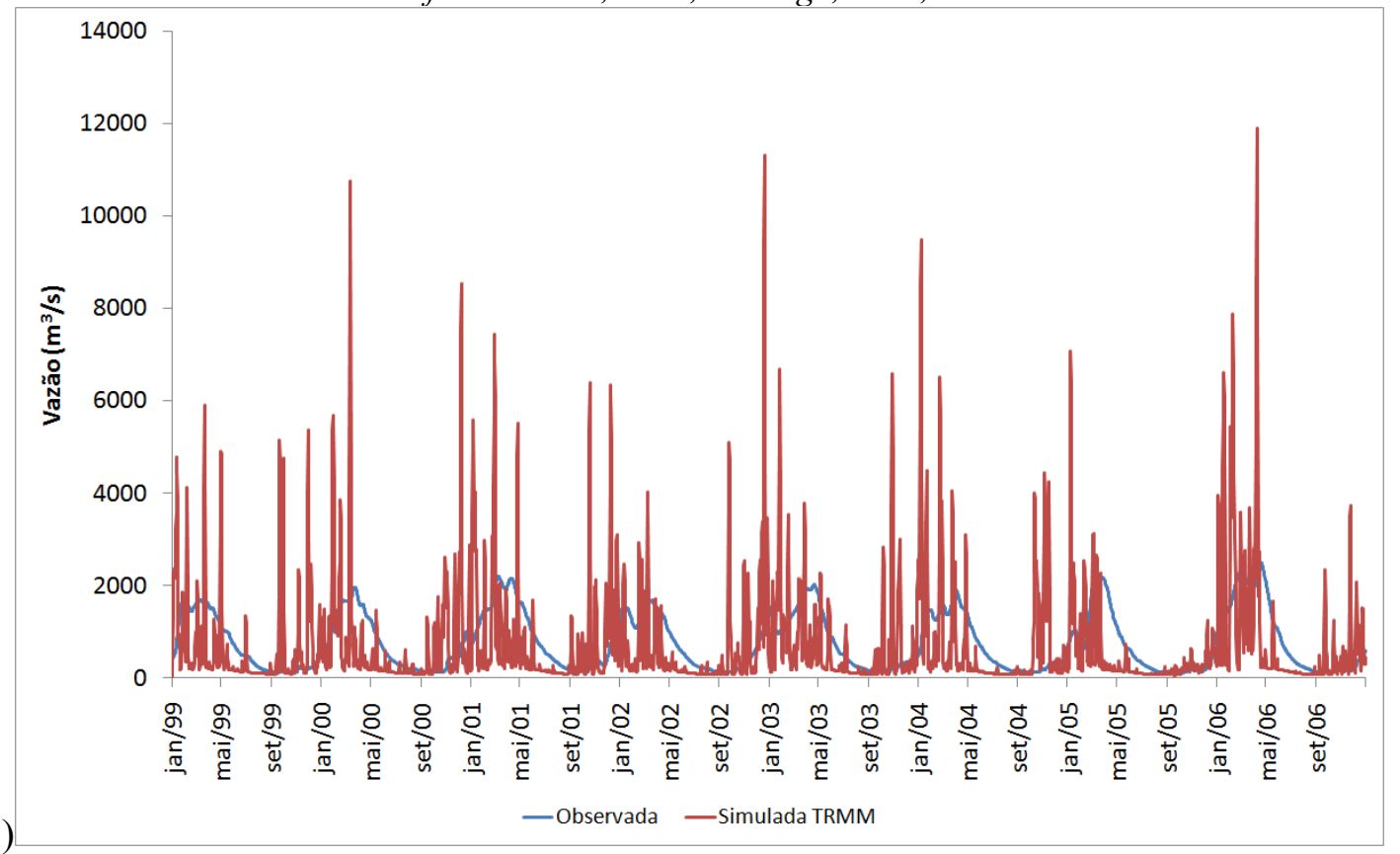

Figura 2 - Hidrogramas de vazões diárias observadas e calculadas na bacia do rio Jamari, em Rondônia, sem parâmetros de calibração

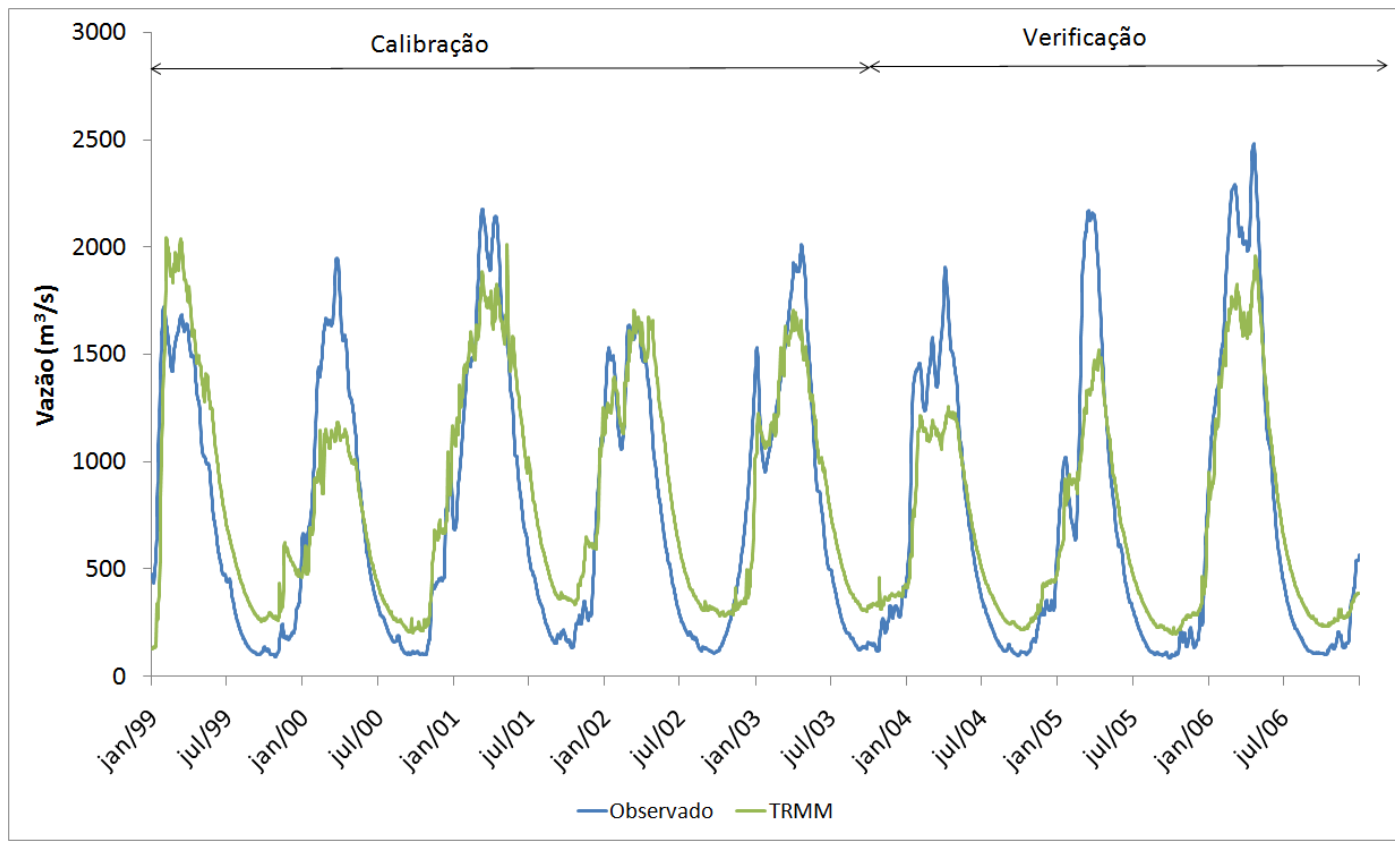

Figura 3 - Hidrogramas de vazões diárias observadas e calculadas na bacia do rio Jamari, em Rondônia, com parâmetros de calibração 
Revista Brasileira de Geografia Física

Recife-PE. V. 01, n. 01, Mai/Ago, 2008, 47-61

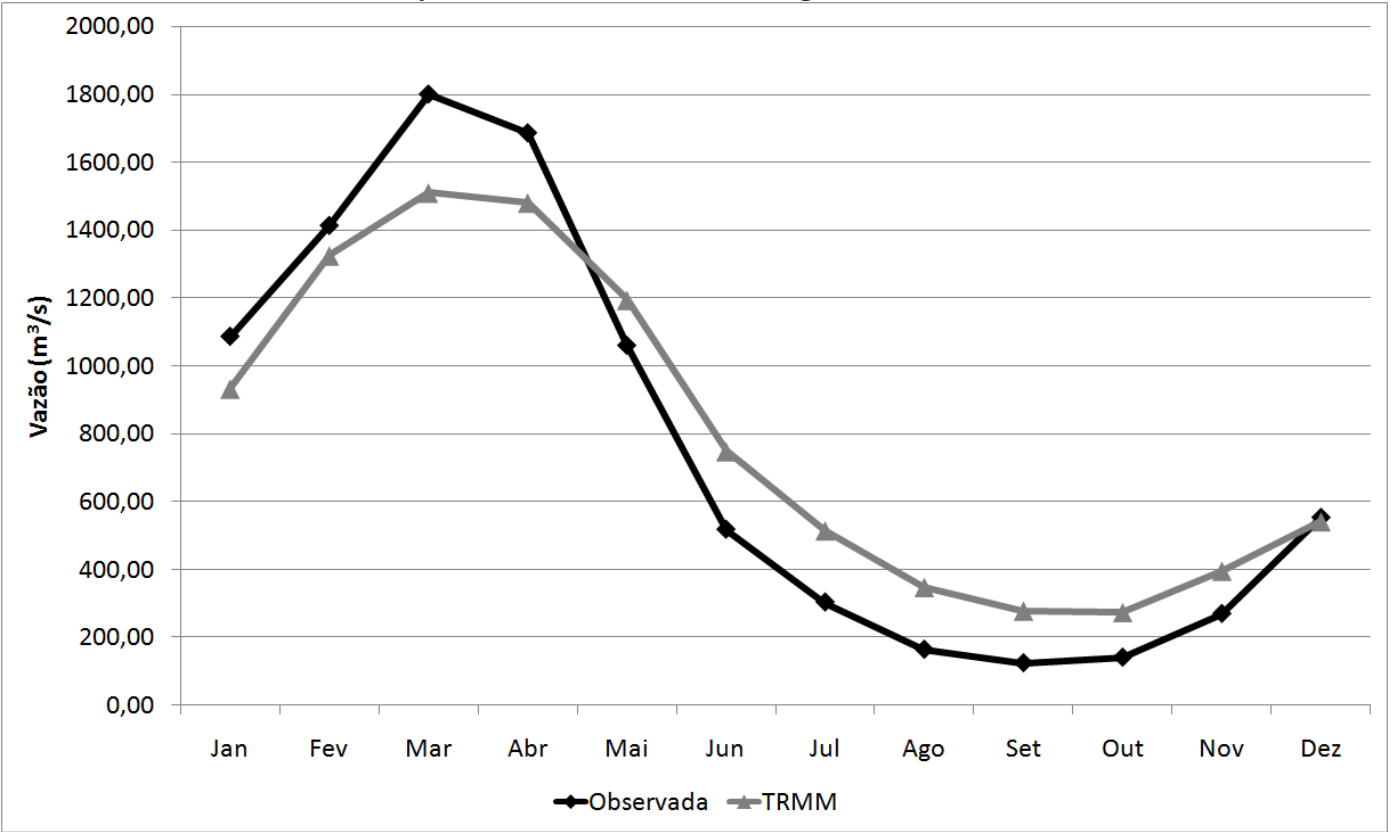

Figura 4 - Hidrograma de vazões médias mensais para o período de 1999 a 2006 observadas e calculadas na bacia do rio Jamari, em Rondônia 